\title{
Necesidad de tratamiento ortodóntico en escolares de 11 a 12 años de la Institución Educativa Mariscal Gamarra del Cusco - Perú, 2019
}

Need for orthodontic treatment in schoolchildren aged 11 to 12 at the Mariscal Gamarra Educational Institution in the city of Cusco - Perú, 2019

Necessidade de tratamento ortodôntico em escolares de 11 a 12 anos na Instituição Educacional Mariscal Gamarra em Cusco - Peru, 2019

\author{
Herbert Cosio Dueñas ${ }^{1}$ ORCID: 0000-0002-9981-7576 \\ Jan Mely Saya Masias² ORCID: 0000-0001-6334-6744 \\ Raquel Mónica Loaiza Carrasco² ORCID: 0000-0003-4097-9076 \\ Liceth Lazo Otazú1 ORCID: 0000-0002-5574-1012
}

\section{Resumen}

Objetivo: Caracterizar la necesidad de tratamiento ortodóntico en su componente de salud dental y estético en escolares de 11 a 12 años de la Institución Educativa Mariscal Gamarra del Cusco - Perú, 2019.

Materiales y métodos: La muestra estuvo constituida por 142 escolares de una institución educativa estatal, se usó el Índice de Necesidad de Tratamiento Ortodóntico (INTO) con sus dos componentes. El Componente de Salud Dental (CDS) es realizado por el Odontólogo y el Componente Estético (CE) es percibido por el paciente.

Resultados: Se obtuvo $15,49 \%$ sin necesidad de tratamiento y $33.10 \%$ de leve necesidad de tratamiento y 28,87\% moderada necesidad de tratamiento en su Componente de Salud Dental y el 74,65\% se presentó sin necesidad de tratamiento en su Componente Estético. Conclusión: Al examen por parte del profesional casi la mitad de los estudiantes no necesitan tratamiento y por otro lado, tres de cuatro estudiantes se perciben sin necesidad de tratamiento de ortodoncia.

Palabras clave: Maloclusión, estética dental, salud bucal (DeCS).

1 Universidad Nacional de San Antonio Abad del Cusco, Cusco, Perú.

2 Universidad Andina del Cusco, Cusco, Perú. 


\section{Abstract}

Objective: To characterize the need for orthodontic treatment in its dental and aesthetic health component in schoolchildren from 11 to 12 years old at the Mariscal Gamarra Educational Institution of Cusco - Peru, 2019.

Materials and methods: The sample consisted of 142 schoolchildren from a public school. The Index of Orthodontic Treatment Need (IOTN) was used with the two components; the Aesthetic Component of Health which was performed by the dentist and the Aesthetic Component which was perceived by the patient.

Results: According to the results, $15.49 \%$ didn't need any treatment, $33.10 \%$ a slight need for treatment, and $28.87 \%$ a moderate need for treatment in its Dental Health component; $74.65 \%$ presented no need for treatment in its Aesthetic component.

Conclusion: Almost half of the students did not need any treatment as a result of the health professional examination. On the other hand; three out of four students showed no need for orthodontic treatment.

Keywords: Malocclusion, dental aesthetics, oral health (DeCS).

\section{Introducción}

Dentro de los problemas de la Salud Pública Bucal, las maloclusiones ocupan el tercer lugar de prevalencia, con un alto porcentaje. En estudios realizados en diferentes latitudes de Latinoamérica se han registrado porcentajes altos de prevalencia en la población. En Cuba se registró el 58,2 \% de estudiantes con algún tipo de maloclusión ${ }^{(1)}$, en Uruguay se registró una prevalencia del $33,8 \%$ en adolescentes y adultos jóvenes ${ }^{(2)}$, en Chile la prevalencia varia del $65 \%$ al $72,6 \%{ }^{(3)}$.

\section{Resumo}

Objetivo: Caracterizar a necessidade de tratamento ortodôntico em seu componente de saúde dental e estética em escolares de 11 a 12 anos de idade na Instituição Educacional Mariscal Gamarra de Cusco - Peru, 2019.

Materiais e métodos: A amostra foi composta por 142 estudantes de uma instituiçáo de ensino estadual, o Índice de Necessidades de Tratamento Ortodôntico (INTO), com seus dois componentes. O componente de saúde bucal (CDS) é realizado pelo dentista e o componente estético (CE) é percebido pelo paciente.

Resultados: foram obtidos $15,49 \%$ sem necessidade de tratamento e $33,10 \%$ para uma leve necessidade de tratamento e $28,87 \%$ para uma necessidade moderada de tratamento em seu Componente de Saúde Bucal e 74,65\% apresentaram sem a necessidade de tratamento em seu Componente Estético.

Conclusão: No exame realizado pelo profissional, quase metade dos estudantes não necessita de tratamento e, por outro lado, três em cada quatro percebem a si mesmos sem a necessidade de tratamento ortodôntico.

Palavras-chave: Má oclusão, estética dental, saúde bucal (DeCS).

El paso de la dentición mixta a la dentición permanente se da lugar entre los diez y los doce años. Es una edad de transición de las últimas piezas temporales y dan lugar las piezas permanentes, en especial los canino, que son los últimos en aparecer en el sector anterior. Por otro lado, la erupción de los segundos molares, que son los últimos en erupcionar en el sector posterior, completando los 28 dientes de la arcada permanente, sin contar a los terceros molares, que serán últimos en hacer aparición en boca ${ }^{(4)}$. La edad de 11 y 12 años es la propicia para po- 
der hacer evaluaciones, de presencia o ausencia de una maloclusión. Por contar a esta edad, con casi todas las piezas en boca ${ }^{(5)}$.

Una maloclusión se puede definir como una desviación de la oclusión ideal o una alteración del estado de normo oclusión; el cual es de origen multifactorial. Muchas de las causas están relacionadas con el aspecto genético y otras tantas con los hábitos o estilos de vida, que el paciente asume mientras desarrolla su oclusión. Se establece que una maloclusión presenta muchas causas, las cuales interactúan entre sí y producen mayor efecto en la desarmonía dentomáxilofacial. Por lo que se hacen necesarios los tratamientos, incluso desde edades muy tempranas, mediante maniobras interceptiva y preventivas ${ }^{(6-7)}$.

En la actualidad se puede apreciar que se ha producido un aumento en la demanda de tratamiento de ortodoncia. Por un lado se presenta la necesidad de tratamiento que clínicamente identifica el profesional; pero también se están dando tratamientos de ortodoncia en función a la demanda por la exigencia estética del paciente, el cual percibe su apariencia estética y plantea al profesional un tratamiento. Este tipo de tratamientos que se dan a raíz de la autopercepción del paciente, se originan por su apariencia dentaria no agradable, por su afectación en la autoestima o porque se presentan dificultades en el desempeño de su vida diaria, es decir el paciente percibe que las maloclusiones afectan su calidad de vida (6). La identificación de los problemas orales se hace por medio de la evaluación clínica del profesional, pero también es importante la participación de los pacientes. Muchos niños manifiestan la necesidad de tratamiento de ortodoncia sobre sus maloclusiones, por lo que el objetivo de este estudio fue caracterizar la necesidad de tratamiento ortodóntico en su componente de salud dental y estético en escolares de 11 a 12 años de la ciudad del Cusco.

\section{Materiales y métodos}

El presente estudio fue descriptivo de corte transversal, aprobado por el comité de bioética de la Universidad Andina del Cusco. La muestra estuvo constituida por 142 nińos de 11 a 12 años de ambos sexos de la Institución Educativa Mariscal Gamarra, quienes reunieron los criterios de selección, el más importante que los padres hayan firmado el consentimiento informado mediante el cual se autoriza la participación del menor en la investigación, así como la participación voluntaria del escolar. A todos se les aplicó el instrumento denominado índice de Necesidad de Tratamiento de Ortodoncia (INTO). El componente estético (CE), que es de carácter subjetivo consistente en una escala análoga visual compuesta por una serie de 10 fotografías intraorales frontales que se corresponden a 10 posibles niveles estéticos dentales. Es importante aclarar que las fotografías corresponden a niños de 12 años con dentición permanente (Fig. 1). La fotografía $\mathrm{N}^{\circ} 1$ se presenta con las características más atractivas y la fotografía $N^{\circ} 10$ se presenta con las características menos atractivas. Este componente fue evaluado por el paciente, según su percepción, reflejando así la necesidad de tratamiento desde el punto de vista social, psicológico del tratamiento de ortodoncia. Se le pidió al niño que identifique la foto de acuerdo a la percepción de su estética dental. Si la foto que elegía se encontrara entre la 1 y la 4 , se consideró sin necesidad de tratamiento; entre la 5 y 7 , con necesidad moderada de tratamiento; y entre la 8 y 10, con necesidad urgente de tratamiento ${ }^{(5,8,9)}$.

Para la recolección de los datos se necesitó la calibración de dos investigadores, los cuales fueron capacitados por un experto. Se obtuvo un coeficiente de concordancia o acuerdo con el estadístico Kappa de Cohen con un valor mayor a 0,8 entre el experto y los investigadores. Los datos obtenidos fueron codificados e ingresados al paquete estadístico SPSS versión 22 en Español. Se usó distribución de frecuencias y porcentajes para el análisis univariado. 

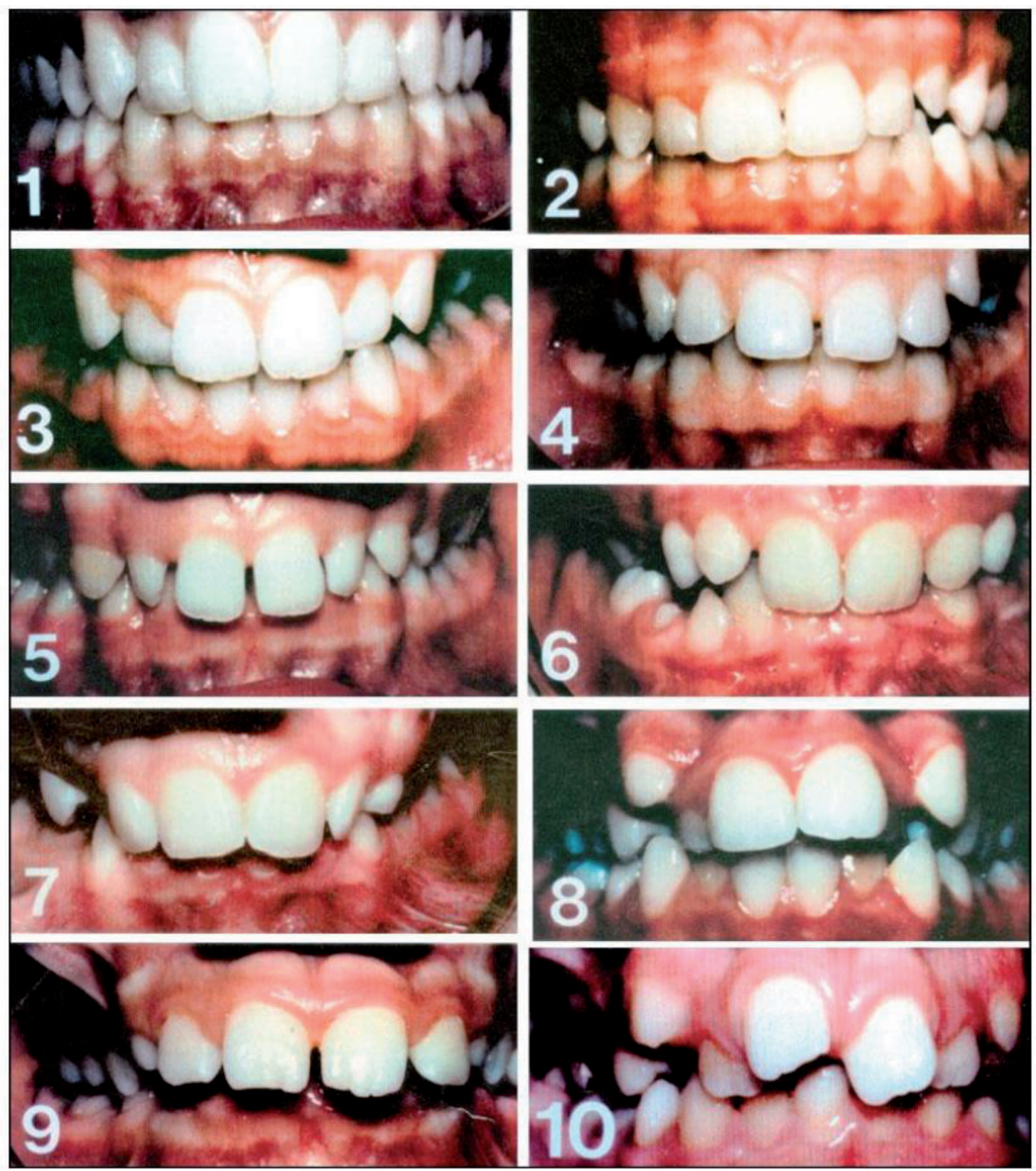

Fig. 1. Fotos del Componente Estético (CE) del INTO

Para el componente de salud oral se realizó un examen clínico en un ambiente adecuado. Se usó la ayuda de una regla milimetrada, se identificó el rasgo más severo al examinar al paciente. Se atribuyó el rasgo más severo para la clasificación del paciente según la necesidad de tratamiento desde el punto de vista objetivo del examinador (Tabla 1). Se observaron las características de los siguientes rasgos oclusales: overjet, overbi- te, mordida abierta, mordida cruzada, erupción impedida, defectos de labio leporino y paladar hendido, anomalías craneofaciales, erupción impedida e hipodoncia. El rasgo oclusal más alterado se utilizó para categorizar la necesidad de tratamiento de ortodoncia desde: sin necesidad de tratamiento (grado 1 y 2), necesidad moderada (grado 3) y con necesidad de tratamiento (grado 4 y 5) ${ }^{(5,8,9)}$. 
Tabla 1. Escalas del Componente de Salud Dental (CSD) del INTO

\section{Grado 5 (Extrema/necesidad de tratamiento)}

5.a Overjet incrementado mayor a $9 \mathrm{~mm}$.

5.h Hipodoncia con implicaciones restaurativas (más de 1 diente perdido en cualquier cuadrante) requiere tratamiento pre-ortodóncico.

5.i Erupción impedida de los dientes (excepto por terceras molares, debido a apiñamiento, desplazamiento, la presencia de supernumerarios, dientes deciduos retenidos y cualquier patología.

5.m Overjet invertido mayor a $3.5 \mathrm{~mm}$ con dificultades masticatorias y del habla.

5.p Paladar y labio fisurado y otras anomalías craneofaciales.

5.s Dientes deciduos sumergidos.

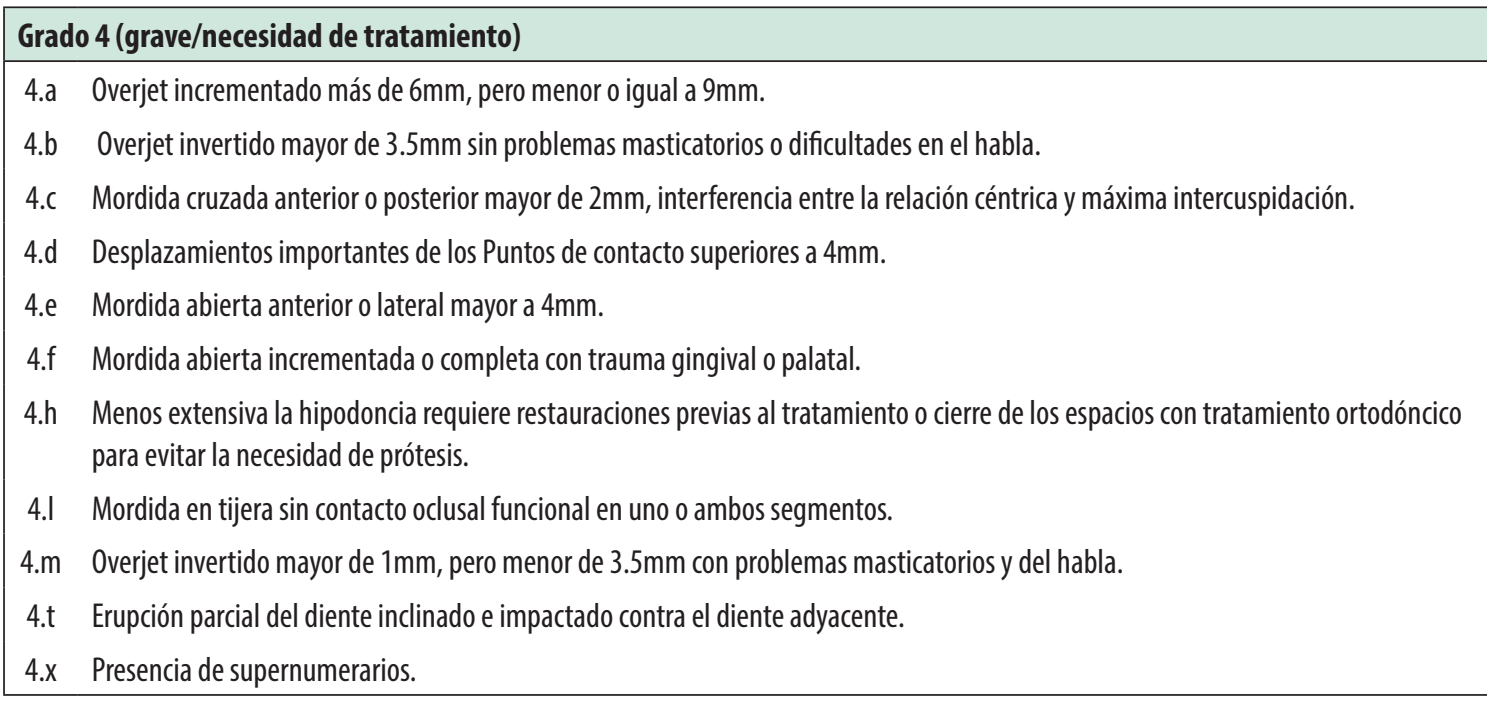

\section{Grado 3 (moderado/límite de necesidad de tratamiento)}

3.a Overjet incrementado mayor de $3.5 \mathrm{~mm}$, pero menor 0 igual a $6 \mathrm{~mm}$.

3.b Overjet invertido mayor a $1 \mathrm{~mm}$, pero menor 0 igual a $3,5 \mathrm{~mm}$.

3.c Mordida cruzada anterior o posterior mayor de $1 \mathrm{~mm}$, pero menor 0 igual a $2 \mathrm{~mm}$. Discrepancia entre oclusión céntrica y máxima intercuspidación.

3.d Desplazamiento de puntos de contacto prematuro superiores a $2 \mathrm{~mm}$, pero inferiores o iguales a $4 \mathrm{~mm}$.

3.e Mordida abierta anterior o lateral mayor de $2 \mathrm{~mm}$, pero menor 0 igual a $4 \mathrm{~mm}$.

3.f Overbite profundo en gingiva o tejido palatal, pero sin trauma.

\section{Grado 2 (leve/sin necesidad de tratamiento)}

2.a Overjet incrementado mayor que $3.5 \mathrm{~mm}$, pero menor 0 igual a $6 \mathrm{~mm}$ con labios funcionales.

2.b Overjet invertido mayor a $0 \mathrm{~mm}$, pero mayor 0 igual a $1 \mathrm{~mm}$.

2.c Mordida cruzada anterior o posterior, menor 0 igual a $1 \mathrm{~mm}$ de discrepancia, entre punto de contacto retrusivo y máxima intercuspidación.

2.d Desplazamiento de los puntos de contacto prematuro superiores a $1 \mathrm{~mm}$, pero inferiores 0 iguales a $2 \mathrm{~mm}$.

2.e Mordida abierta anterior 0 posterior mayor de $1 \mathrm{~mm}$, pero menor 0 igual a $2 \mathrm{~mm}$.

2.f Overbite incrementado mayor o igual a $3.5 \mathrm{~mm}$ sin contacto gingival.

2.g Oclusión pre-normal o post-normal sin otras anormalidades (incluyen hasta la mitad de una unidad de discrepancia). 


\section{Resultados}

De la muestra de 142 niños el 12,7\% fueron niños de 11 años y el 87,3\% de 12 años. Con respecto al sexo el $54,2 \%$ fueron nińos y el $45,8 \%$ niñas (Tabla 2).

Con respecto al Componente de Salud Dental el grado de necesidad de tratamiento de ortodoncia fue de $15,5 \%$ grado 1 , sin necesidad. El 33,1\% fue identificado como grado 2, leve necesidad de tratamiento. El 28,9\% fue identificado como grado 3, moderada necesidad de tratamiento. Es decir el 77,5\% de los evaluados se encontraron dentro del límite de necesidad de tratamiento, el resto mostro grave y extrema necesidad de tratamiento de Ortodoncia (Tabla 3).

Con respecto al Componente Estético el grado de necesidad de tratamiento de ortodoncia percibido por el escolar fue de $74,6 \%$ sin necesidad de tratamiento. El 15,5\% se identificó con moderada necesidad de tratamiento. El 9,9\% se identificó con necesidad de tratamiento de ortodoncia (Tabla 4).

Tabla 2. Distribución de la edad y sexo de los niños de 11 y 12 años.

\begin{tabular}{|c|c|c|c|c|c|}
\hline & & & \multicolumn{2}{|c|}{ Sexo del niño } & \multirow{2}{*}{ Total } \\
\hline & & & Masculino & Femenino & \\
\hline \multirow[t]{4}{*}{ Edad del niño } & \multirow[t]{2}{*}{11 años } & $\mathrm{N}$ & 11 & 7 & 18 \\
\hline & & $\%$ & $7,7 \%$ & $4,9 \%$ & $12,7 \%$ \\
\hline & \multirow[t]{2}{*}{12 años } & N & 66 & 58 & 124 \\
\hline & & $\%$ & $46,5 \%$ & $40,8 \%$ & $87,3 \%$ \\
\hline \multirow{2}{*}{\multicolumn{2}{|c|}{ Total }} & $\mathrm{N}$ & 77 & 65 & 142 \\
\hline & & $\%$ & $54,2 \%$ & $45,8 \%$ & $100,0 \%$ \\
\hline
\end{tabular}

Tabla 3. Componente de Salud Dental (CSD) del INTO.

\begin{tabular}{|l|c|c|c|}
\hline & Frecuencia & Porcentaje & Porcentaje acumulado \\
\hline Grado 1 (sin necesidad de tratamiento) & 22 & $15,5 \%$ & $15,5 \%$ \\
\hline Grado 2 (leve/sin necesidad de tratamiento) & 47 & $33,1 \%$ & $48,6 \%$ \\
\hline Grado 3 (moderado de necesidad de tratamiento) & 41 & $28,9 \%$ & $77,5 \%$ \\
\hline Grado 4 (grave/necesidad de tratamiento) & 30 & $21,1 \%$ & $98,6 \%$ \\
\hline Grado 5 (Extrema/necesidad de tratamiento) & 2 & $1,4 \%$ & $100,0 \%$ \\
\hline Total & 142 & $100,0 \%$ & \\
\hline
\end{tabular}

Tabla 4. Componente Estético (CE) del INTO.

\begin{tabular}{|l|c|c|c|}
\hline & Frecuencia & Porcentaje & Porcentaje acumulado \\
\hline Sin necesidad de tratamiento (fotografías entre 1 a 4) & 106 & $74,6 \%$ & $74,6 \%$ \\
\hline Con necesidad moderada (fotografías entre 5 a 7) & 22 & $15,5 \%$ & $90,1 \%$ \\
\hline Con necesidad de tratamiento (fotografías entre 8 a 10) & 14 & $9,9 \%$ & $100,0 \%$ \\
\hline Total & 142 & $100,0 \%$ & \\
\hline
\end{tabular}




\section{Discusión}

Se evaluó la necesidad de tratamiento de Ortodoncia mediante el Índice creado por Brook y Shaw, desarrollado en el Reino Unido, el cual tiene dos componentes: uno objetivo, el cual es elaborado por el clínico sobre la salud dental del paciente y el otro componente subjetivo, donde el paciente percibe las alteraciones estéticas de su maloclusión.

El análisis de las diferentes características oclusales en forma clínica y haciendo el registro de las alteraciones por parte del profesional, teniendo en consideración una escala de medición previamente establecida, se denomina el Componente de Salud Dental del Índice de Necesidad de Tratamiento de Ortodoncia, hemos podido observar un porcentaje 33,1\% de leve, 28,9\% moderada y $21,1 \%$ de grave necesidad de tratamiento de ortodoncia, componente identificado por el profesional al momento de hacer la evaluación clínica. El Componente de Salud Dental se basó en el análisis de distintas características oclusales por parte del profesional, y el registro de las alteraciones de la misma, dentro de una escala. Al analizar los resultados que nos arroja el Componente de Salud Dental, podemos manifestar que es más confiables, porque es realizado por el Odontólogo sobre un análisis objetivo de las condiciones de salud oral del escolar, quien a los 12 años ya presenta todas las piezas dentales permanentes erupcionadas, en quien se puede apreciar la presencia de maloclusiones dentales y esqueléticas. Se puede hacer el diagnóstico sin temor a equivocarse.

La autopercepción de la estética para establecer la necesidad de tratamiento de ortodoncia a través de la selección de una fotografía, se denomina Componente Estético del Índice de Necesidad de Tratamiento de Ortodoncia.

A través de esto se encontró que el 74,6\% de los escolares percibió que no necesitaban tratamiento de ortodoncia. Esta percepción ha sido definida por el estudiante sobre su estética dental, de una serie de 10 fotografías intraorales frontales, ; este porcentaje alto se podría deber a una alta autoestima del estudiante, quien a pesar de tener una moderada necesidad de tratamiento, percibía que no necesitaba ser tratado por el Ortodoncista. En cambio los resultados que nos arroja el Componente estético, a nuestro parecer es menos confiables porque se trata de una apreciación subjetiva de un escolar de 12 años, que todavía no ha entrado a un estado de madurez emocional, que le permita ser autocrítico sobre su estética dental; son preadolescentes en quienes el desarrollo de su personalidad está en proceso. Muchos jóvenes a esta edad todavía no se preocupan mucho por su estética, a pesar de presentar un problema clínico de manifiesto. Al comparar nuestros resultados con otras investigaciones se pudo ver que en 170 escolares de 11 a 12 ańos en una escuela privada en Ecuador, el 91,18\% percibió que no necesitaba tratamiento de ortodoncia, según el componente estético de salud. Por otro lado en el $10 \%$ se identificó sin necesidad de tratamiento, según el componente de Salud Dental (5). Así mismo, en 143 escolares de 12 años en Cuenca se pudo ver que $82 \%$ no percibió necesidad de tratamiento de ortodoncia, el 13\% una moderada y el 5\% una necesidad severa de tratamiento ${ }^{(10)}$. Se evaluó a 140 escolares de 12 años en Cuenca Ecuador, el $81 \%$ tuvo leve necesidad de tratamiento, $14 \%$ moderada necesidad y $6 \%$ grave necesidad de tratamiento de ortodoncia autopercibida por el escolar ${ }^{(9)}$. En un estudio realizado sobre 166 modelos de estudio pretratamiento de ortodoncia, el 65\% se identificó que no tenía necesidad de tratamiento, el 9,03\% necesidad moderada y el $25,3 \%$ una gran necesidad de tratamiento ${ }^{(7)}$. Todos estos resultados que difieren a lo hallado en una población de 76 escolares Aymaras que viven en el altiplano Chileno, en quienes se encontró $71 \%$ de necesidad de tratamiento de ortodoncia en el CSD del INTO, habiendo sido identificados dentro del grado 4 y 5 . Estos resultados se muestran diferentes, tal vez por las características del biotipo del poblador Aymara ${ }^{(3)}$. Así mismo, en una población adolescente de 12 a 15 años en la ciudad de Viña del Mar en Chile, se registró $44,2 \%$ de necesidad de tratamiento en su componente estético, porcentaje alto quizás por ser un grupo con una mayor edad, es posible 
que a los 15 años ya se haya logrado una madurez emocional suficiente y que permita al adolecente ser más objetivo al momento de establecer su percepción sobre la necesidad de tratamiento de ortodoncia ${ }^{(13)}$. En 200 pacientes con un rango de edad de 13 a 25 años de edad, el 66\% presentó un grado 4 , es decir grave necesidad de tratamiento. Dicho resultado se puede deber a la edad muy avanzada de los evaluados ${ }^{(14)}$. En 210 escolares de 12 a 16 años de edad, en Lima se encontró que el 73,33\% estaban en necesidad de tratamiento, el 19,52\% en necesidad moderada y 7,14 sin necesidad de tratamiento en el componente de salud dental; así mismo, el 83,3\% se percibían sin necesidad de tratamiento, el 14,3\% con necesidad moderada y el $2,4 \%$ con necesidad definitiva de tratamiento de ortodoncia en su componente estético ${ }^{(11)}$.

\section{Conclusiones}

Con respecto al índice de necesidad de tratamiento ortodóncico en su componente de salud dental, la mitad de los escolares de 11 a 12 ańos tiene moderada, grave y extrema necesidad de tratamiento de ortodoncia, resultados que difieren con respecto al índice de necesidad de tratamiento ortodóncico en su componente estético, ya que una cuarta parte de los escolares de 11 a 12 ańos perciben una necesidad moderada y alta necesidad de tratamiento de ortodoncia.

\section{Contribución de autoría}

1. Concepción y diseño del estudio

2. Adquisición de datos

3. Análisis de datos

4. Discusión de los resultados

5. Redacción del manuscrito

6. Aprobación de la versión final del manuscrito

HCD ha contribuido en 1, 3, 4, 5, 6 .

JMSM ha contribuido en 1, 2, 3, 4 .

RMLC ha contribuido en 5, 6 .

LLO ha contribuido en 4, 5, 6, 7 .

\section{Referencias}

1. Pino IM, Veliz OL, García PA. Maloclusiones, según el índice de estética dental, en estudiantes de séptimo grado de Santa Clara. Medicentro Electrónica. 2014; 18 (4): 177-9.

2. Ourens M, Celeste R, Lorenzo S, Neves H, Alvarez R, Abegg C. Prevalencia de maloclusiones en adolescentes y adultos jóvenes del interior del Uruguay. Relevamiento nacional de salud bucal 2010-201. Odontoestomatología. 2013; 15 (1): 47-57.

3. Segeur K, Fuentes F, Sabando V, Donaire F, Vásquez A. Prevalencia de Maloclusión y Caries Dental en Niños Aymaras de Colchane, Chile. Inter J of odont. 2020; 14 (2): 191-7.

4. Graber LW, Vanarsdall RL, Vig KW. Ortodoncia - Principios y técnicas actuales. 5ta ed. Barcelona España: ELSEVIER; 2013.

5. Crespo $\mathrm{C}$, Domínguez $\mathrm{C}$, Vallejo $\mathrm{F}$, Liñán $\mathrm{C}$, Del Castillo C, León-Manco R A, Evangelista A. Impacto de maloclusiones sobre la calidad de vida y necesidad de tratamiento ortodóntico en escolares de dos escuelas privadas Azogues - Ecuador, 2015. Rev. Estomatol. Herediana. 2017; 27 (3): 141.

6. Castillo M, Mejías O. Necesidad de tratamiento ortodóncico según el índice IOTN en niños escolares de la unidad educativa "Maribel Caballero de Tirado". Rev Venez. Invest IADR. 2016; 4 (1): 40-9.

7. Cruz MF, Gutiérrez MF, Gutiérrez JF, Rojas AR. Comparación de los índices ICON y el componente estético del IOTN para determinar la necesidad de tratamiento ortodóncico. Revista Mexicana de Ortodoncia. 2017; 5 (1): 11-4.

8. Rodriguez JE. Prevalencia de maloclusión y necesidad de tratamiento ortodóntico en escolares de 12 años de instituciones educativas públicas - Trujillo 2015 [tesis]. [Trujillo Perú]: Universidad Nacional de Trujillo; 2016.

9. Zhañay JE. Relación entre autopercepción de Necesidad de Tratamiento Ortodoncico y la Prevalencia de maloclusiones en pacientes de 12 años en la parroquia el Sagrario [tesis]. [Cuenca Ecuador]: Universidad Católica de Cuenca; 2016.

10. Guerrero J, Villavicencio E, González L. Autopercepción de necesidad de tratamiento orto- 
dóntico en escolares de 12 años en la Parroquia El Sagrario del Cantón Cuenca-Ecuador. Revista Estomatológica Herediana. 2017; 27 (2): 6773.

11. Pardo K. Índice de Necesidad de tratamiento ortodóntico en escolares del nivel de secundaria de la I. E. N 154 "Carlos Noriega Jimenez" [tesis]. [Lima Perú]: Universidad San Martin de Porres; 2012.

12. Manccini LM. Necesidad de tratamiento ortodóntico según el índice de necesidad de tra- tamiento ortodóntico en escolares de 12 a 13 años [tesis]. [Lima Perú]: Universidad Nacional Mayor de San Marcos; 2011.

13. Cueto A, Skog F, Muńoz M, Espinoza S, Muńoz D, Martínez D. Prevalencia de Anomalías Dentomaxilares y Necesidad de Tratamiento en Adolescentes. International journal of odontostomatology. 2017; 11 (3): 333-8.

14. Avilés M, Huitzil E, Fernández M, Vierna JM. Índice de necesidad de tratamiento ortodóncico (IOTN). Revista Oral. 2011; 12 (39): 782-5.

Herbert Cosio Dueñas: hcosiod@hotmail.com 